\title{
475384 - SHEAR RATES DERIVED FROM ULTRASOUND IMAGES AND PARTICLE IMAGE VELOCIMETRY
}

\author{
Clifton Johnston, $\mathbf{P h D}^{2}$, Melissa Lui, B. Eng (in progress) ${ }^{2}$, Kogan Lee, BEng (in \\ progress) $^{2}$, Andrew Walker, BSc, MBIS ${ }^{1}$, Gary Dobson, M.D.,C.M. ${ }^{1}$ \\ 1. Anesthesia, University of Calgary, Calgary, AB, Canada \\ 2. Mechanical Engineering, University of Calgary, Calgary, AB, Canada
}

Introduction: Shear stress is a primary determinant of endothelial cell function(1). Current ultrasound approximations of shear stress have limited spatial resolution due to the large sample volume of Doppler based techniques(2). Particle Image Velocimetry (PIV) can be applied to ultrasound derived images and may be used to determine shear stress.

Methods: Latex tubing $(1 / 2 \mathrm{~cm}$. diameter) was suspended in a custom-made plexiglass chamber with an acoustic window placed to allow for ultrasound interrogation. The chamber and tubing were filled with normal saline and flow through the tubing driven by a commercial fluid pump. Ultrasound images were acquired with a linear probe at 10 $\mathrm{mHz}$ and a frame rate of 78. Echo-contrast was added to the normal saline until images appropriate for analysis with PIV were obtained. Recordings were made during steady and pulsatile flow. Mid-tubing velocity was determined by pulse wave Doppler to provide a standard for comparison, and to allow for the calculation of the theoretical velocity profiles.

Results: The velocity profile of the flow was measured using a proprietary PIV program. During steady flow, the Echo-PIV derived velocities corresponded closely to the theoretical velocities calculated from the pulse wave Doppler (Figure 1). Maximum velocity at the mid-tube, as represented by the scaled vector, was $7.5 \mathrm{~cm} / \mathrm{s}$. Similar results were obtained during pulsatile flow, with the predicted flow reversal occurring along the outer walls.

Discussion: PIV represents an improved means of quantifying fluid velocity profiles, and consequently shear stress. PIV velocity is not dependent upon the angle of interrogation and its spatial resolution is not limited by the sample volume. PIV can also be used to quantify flow characteristics, such as vortices, that are not readily amenable to standard ultrasound techniques. Clinical measurements of shear rates using this technique are currently limited to diastole flow due to the low frame rates of commercial ultrasound machines.

References: (1) Mitchell GF, Parise H, Vita JA, Larson MG, Warner E, Keaney JF, Jr., et al. Local shear stress and brachial artery flow-mediated dilation: the Framingham Heart Study. Hypertension 44(2):134-9, 2004 Aug. (2)Tortoli P, Bambi G, Guidi F, Muchada R. Toward a better quantitative measurement of aortic flow. Ultrasound in Medicine \& Biology 28(2):249-57, 2002 Feb. 


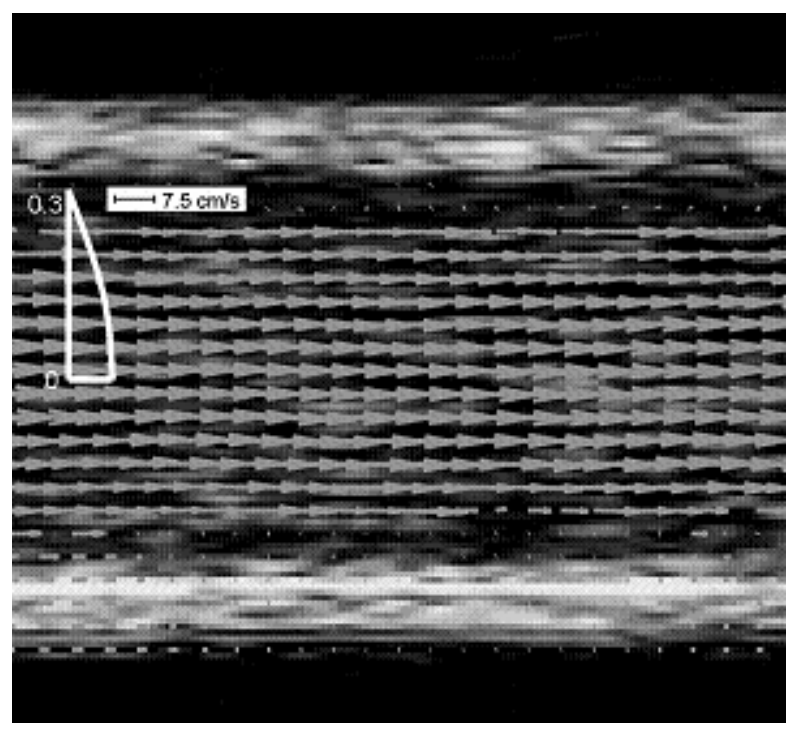

